\title{
La evaluación de la alimentación humana según maestros en formación de España/Portugal
}

\author{
The evaluation of human feeding acordin to training teachers from \\ Spain/Portugal
}

\author{
Juan Carlos Rivadulla-López, María-Jesús Fuentes-Silveira, Susana García Barros \\ Universidade da Coruña
}

\begin{abstract}
Resumen
En este trabajo se analizan las cuestiones de evaluación propuestas por maestros en formación de la Universidade da Coruña (España) y Universidade do Minho (Portugal) para $5 \% 6^{\circ}$ de Educación Primaria sobre la alimentación y la salud para conocer los contenidos evaluados y las habilidades exigidas en ellas. La recogida de datos se realizó mediante una actividad formativa de aula. Los resultados muestran que aunque existen diferencias entre ambos colectivos tienen una idea restringida de los contenidos que deben ser evaluados, lo que constituye una llamada de atención para los sistemas formativos que se vienen empleando.

Palabras clave: Educación Primaria, formación inicial, evaluación, habilidades, alimentación humana.
\end{abstract}

\begin{abstract}
This paper analyzes the evaluation questions proposed by teachers in training at the Universidade da Coruña (Spain) and Universidade do Minho (Portugal) for $5^{\circ} / 6^{\circ}$ of Primary Education on food and health in order to know the contents evaluated and the skills required in them. Data collection was done through a classroom training activity. The results show that although there are differences between both groups they have a restricted idea of the contents that must be evaluated, which constitutes a wake-up call for the training systems that are being used. Keywords: Primary Education, initial training, evaluation, skills, human feeding.
\end{abstract}

\section{Introducción}

Los conocimientos científicos que se trabajan en las aulas se consideran importante cuando es objeto de evaluación (Goytia, Besson, Gasco, \& Domènech, 2015). En este sentido, la evaluación ha sido entendida tradicionalmente por el profesorado como una "valoración" (López-Lozano \& Solís-Ramírez, 2016), que sirve para detectar los puntos fuertes y débiles de los conocimientos del alumnado (Wang, Kao, \& Lin, 2009), para informar al alumnado y a sus familias al respecto y para recuperar, así, los aprendizajes no adquiridos (Hernández, 2010). Además, la mayoría del profesorado a la hora de evaluar a sus alumnos cree y confía en la objetividad de los instrumentos tradicionales, es decir, del examen escrito (Miralles, Gómez, \& Sánchez, 2014).
Sin embargo, algunos docentes ya consideran el carácter procesual de la evaluación (Solís-Ramírez, 2012) y utilizan un conjunto combinado de estrategias, reconociendo que ello mejora su rendimiento académico (Weurlander, Söderberg, Scheja, Hult, \& Wernerson, 2012). De hecho se admite que el sistema de evaluación usado por cada profesor determina la calidad del proceso de enseñanza y la mejora del aprendizaje (Brown, Lake y Matters, 2011). Para ello, la observación del trabajo en clase es el procedimiento, técnica e instrumento que más valora el profesorado a la hora de evaluar a sus alumnos/as (Blanco Loro, 2010).

Por otra parte, los profesores tienen que tomar decisiones fundamentadas $\mathrm{y}$ reflexivas sobre los contenidos que deben impartirse en el aula, lo cual no siempre es fácil. Así, el presente trabajo toma como referente el análisis científico / didáctico de la alimentación humana que ha de enseñarse en la Educación Primaria. Su elección se debe a que es un tópico científico que se incluye como objeto de aprendizaje reiterado a lo largo de la educación obligatoria, sobre el que los alumnos suelen tener conocimientos restringidos (Núñez, Mazzitelli, \& Vázquez, 2007) o mantener hábitos inadecuados (Rivarosa \& De Longhi, 2006; Banet \& López, 2010; Vidgen, 2016) planteándose su estudio de forma progresiva, es decir, con un nivel de profundidad creciente. Es conveniente que el alumnado parta de un conocimiento elemental/descriptivo del significado de la dieta saludable, asociada a entender que es necesario ingerir alimentos variados o en un nivel mayor de concreción, a conocer los diferentes tipos de nutrientes (glúcidos, lípidos, proteínas) en las proporciones adecuadas (Rivadulla López, García Barros, \& Martínez Losada, 2013). Pero, además de conocer cómo tiene que ser una dieta saludable, el alumnado también tiene que saber justificar por qué es saludable (Cabello, España, \& Blanco, 2016), conociendo los alimentos que se deben incluir en la dieta y conocer que muchas enfermedades que padecemos están relacionadas con hábitos inadecuados de alimentación (Del Carmen, 2010). Además, los hábitos saludables respecto a la alimentación trascienden a la misma, siendo necesario 
contemplar otros aspectos no menos importantes (higiene, ejercicio físico...) (Pérez de Eulate \& Llorente, 2015).

Por otro lado, no podemos olvidar que desde la perspectiva de la importancia que tiene actualmente el uso del lenguaje en las clases de Ciencias, en la medida que permite expresar y organizar ideas (Prain, 2006), resulta imprescindible que los docentes exijan diferentes tipos de habilidades implicadas en el aprendizaje científico, en sus cuestiones de evaluación tanto de tipo cognitivo como cognitivo-lingüístico (Jorba, Gómez, \& Prat, 2000). Entre estas últimas cabe destacar la descripción de hechos, la definición de conceptos y la justificación de determinados hechos empleando un marco teórico de referencia. En este sentido, se han detectado deficiencias entre el profesorado, tanto en ejercicio como en formación (Martínez Losada \& García Barros, 2013; García Barros \& Martínez Losada, 2014).

Por todo ello, en este trabajo pretendemos describir cómo conciben la evaluación de la alimentación humana un grupo de futuros docentes de Educación Primaria de España y Portugal, tanto desde el punto de vista conceptual (qué contenidos evaluarían) como procedimental (qué habilidades incluyen las cuestiones de evaluación). Es cierto que existen algunos estudios que se centran en comparar las creencias del profesorado de diferentes países (Titrek \& Cobern, 2011; Briceño, Benarroch, \& Marín, 2013), pero a pesar de que este tipo de trabajos ofrecen buenas oportunidades para explorar la interacción entre conocimientos científicos y contexto cultural, se puede decir que hay poca investigación realizada con profesorado de ciencias (Young \& Nehm, 2011). Y, concretamente, no se ha encontrado ningún trabajo comparativo sobre el pensamiento docente acerca de enseñanza de la alimentación humana.

\section{Método}

Teniendo en cuenta la finalidad del estudio se optó por un enfoque metodológico cualitativo (Sabariego, Massot, $\&$ Dorio, 2012) mediante una actividad realizada por los maestros en formación en el marco de las asignaturas de Didáctica de las Ciencias Experimentales. Esta actividad se realizó en el horario habitual de clase.

A los participantes se les indica que la actividad que van a realizar, además de servir cómo actividad formativa, va a formar parte de una investigación y, para ello, se les pide su consentimiento, garantizándoles la confidencialidad de los datos aportados. Durante su realización se promueve un ambiente cordial y distendido. Las actividades se recogieron en papel, para posteriormente proceder a su análisis.

\section{Participantes}

En este estudio han participado por un lado 29 pequeños grupos de dos/tres maestros en formación de $3^{\circ}$ curso de la Diplomatura de Maestro de Educación Primaria en la Universidade da Coruña (España). Por otro lado han participado 19 pequeños grupos de tercer curso de la Licenciatura en Educación Básica de la Universidade do Minho (Portugal).

Esta muestra resulta coherente con la investigación cualitativa, pues responde a la accesibilidad de los participantes en el momento de la realización de la misma (McMillanm \& Shumacher, 2005), y su número es suficiente, ya que en este tipo de metodología no es factible ni necesaria una muestra amplia (Stake, 2007).

\section{Instrumentos o Materiales}

Como instrumento de recogida de datos se utilizaron las propias actividades que realizan los futuros maestros durante su etapa formativa en el marco de la asignatura de Didáctica de las Ciencias Experimentales de cada universidad. Se trataba de una actividad de aplicación en la que debían elaborar instrumentos específicos con objeto de evaluar el aprendizaje de unos hipotéticos alumnos de $5^{\circ}$ y $6^{\circ}$ de Educación Primaria en relación a la nutrición humana. Cabe destacar que este alumnado recibió formación específica sobre la nutrición humana en las diversas asignaturas relacionadas con la Didáctica de las Ciencias Experimentales a lo largo de la Diplomatura en España y de la Licenciatura en Portugal.

\section{Procedimiento}

Los futuros docentes plantearon cuestiones de evaluación referidas a distintos aspectos relacionados con la nutrición humana. Cada cuestión puede incluir preguntas referidas a más de un aspecto o habilidad. Para analizarlas, se organizaron en torno a cuatro dimensiones relativas a este tópico: a) el concepto y finalidad de la nutrición, b) los cuatro sistemas que intervienen en la nutrición humana, c) la alimentación y la salud, y d) la interrelación entre la alimentación/nutrición humana y el medio.

En esta ocasión nos centraremos únicamente en el estudio de las cuestiones referidas a la dimensión sobre la alimentación y la salud, que fueron formuladas por un total de 21 grupos en el caso de España y de 19 en el caso de Portugal. Concretamente, el colectivo español planteó un total de 40 cuestiones asociadas a este tema, mientras que el portugués 60 .

Para analizar las cuestiones planteadas, dentro de esta dimensión se establecieron categorías que responden a distintas ideas clave: A1: Alimentación variada y salud (aquella que nos ayuda a mantenernos sanos); A2: Características de la alimentación saludable (la alimentación aporta distintos tipos de nutrientes que nuestro organismo necesita); A3: Relación entre alimentación y nutrición (una buena alimentación favorece una adecuada nutrición); A4: Hábitos saludables (hábitos y enfermedades relacionadas con la alimentación/nutrición) y A5: Otros aspectos (origen y cantidad de alimentos...). A su vez, respecto a cada pregunta se identificaron las habilidades cognitivas y cognitivo lingüísticas que incluyen. Dentro de estas últimas estarían aquellas que se ponen en marcha para analizar y comprender la información recibida y que se expresan oralmente o por escrito (Ds -Descripción-, DfDefinición-, Ex -Explicación- y J -Justificación-...), mientras que dentro de las cognitivas estarían las que no requieren expresarse ni oralmente ni por escrito (Ic Identificación de características- y Er -Establecimiento de relaciones-).

En primer lugar se analizaron las aportaciones de forma global, tanto en lo que se refiere a las ideas clave, como a 
las habilidades que incluyen en las cuestiones planteadas. A continuación se realizó un análisis individualizado de dichas cuestiones, con objeto de identificar cada idea clave con las habilidades que se asocian a cada una de ellas.

\section{Resultados}

El análisis global de las aportaciones que realizan los 21 grupos españoles en relación a la alimentación (Figura 1), muestra que la gran mayoría (16 grupos) plantea cuestiones relativas a las características la alimentación saludable (idea clave A2). Además, 3 grupos incluyen alguna cuestión sobre la relación entre alimentación y salud (idea clave A1) y 5 lo hacen sobre hábitos saludables en relación a la alimentación (idea clave A4). Ningún participante plantea cuestiones referidas a la relación entre alimentación y nutrición (A3).

El colectivo portugués se centra sobre todo en las ideas clave A2 y A4) (14 grupos en cada caso). Además, 5 grupos se refieren a la idea clave A1, 4 a la A3 y 1 a la A5.

Por otra parte, la mayoría de los participantes solicitan en sus cuestiones el uso de habilidades cognitivo-lingüísticas, sobre todo la descripción (12 grupos españoles y 12 portugueses) y en menor medida la explicación y la justificación (3 y 4 grupos españoles respectivamente y 2 y 4 portugueses). Además, 7 grupos españoles y 12 portugueses solicitan habilidades cognitivas, concretamente la identificación de características.



Figura $1 . \mathrm{N}^{\mathrm{o}}$ de futuros maestros que tienen en cuenta las distintas ideas clave y habilidades en las cuestiones de evaluación planteadas sobre alimentación y salud.

Un análisis más detallado de las aportaciones que realiza cada grupo muestra que la mayoría de los grupos españoles atienden a un solo tipo de idea clave (15 grupos) mientras que los portugueses suelen atender a dos tipos (11 grupos). Los demás grupos españoles (6) consideran dos ideas clave, centrándose mayoritariamente en las características de la alimentación saludable (A2), mientras que los portugueses, además de centrarse en un tipo (4 grupos) también lo hacen en 3 tipos de ideas clave (4 grupos), predominando también la idea clave A2.
Tabla 1.

$N^{o}$ de ideas clave que cada grupo de maestros en formación incluye en las cuestiones de evaluación sobre alimentación y salud.

\begin{tabular}{|c|c|c|c|c|c|}
\hline \multicolumn{2}{|c|}{ Idea clave } & \multicolumn{2}{|c|}{$\begin{array}{c}\text { España } \\
(n=21)\end{array}$} & \multicolumn{2}{|c|}{$\begin{array}{c}\text { Portugal } \\
(n=19)\end{array}$} \\
\hline \multirow{4}{*}{ Un tipo } & A1 & 2 & \multirow{4}{*}{15} & -- & \multirow{4}{*}{4} \\
\hline & $\mathrm{A} 2$ & 11 & & 3 & \\
\hline & A4 & -- & & 1 & \\
\hline & $\mathrm{A} 5$ & 2 & & -- & \\
\hline \multirow{4}{*}{ Dos tipos } & $\mathrm{A} 2+\mathrm{A} 4$ & 4 & \multirow{4}{*}{6} & 7 & \multirow{4}{*}{11} \\
\hline & $\mathrm{A} 2+\mathrm{A} 5$ & 1 & & -- & \\
\hline & $\mathrm{A} 2+\mathrm{A} 3$ & -- & & 2 & \\
\hline & $\mathrm{A} 1+\mathrm{A} 4$ & 1 & & 2 & \\
\hline \multirow{2}{*}{ Tres tipos } & $\mathrm{A} 1+\mathrm{A} 3+\mathrm{A} 4$ & -- & \multirow[b]{2}{*}{-} & 2 & \multirow{2}{*}{4} \\
\hline & $\mathrm{A} 2+\mathrm{A} 4+\mathrm{A} 5$ & -- & & 2 & \\
\hline
\end{tabular}

En cuanto al tipo de habilidades que exigen en las cuestiones, 17 grupos de españoles se centran en una, siendo la descripción (Ds) la más frecuente (9 grupos), mientras que los portugueses oscilan entre un tipo de habilidad (9 grupos) centrándose sobre todo en la identificación de características (Ic) y dos tipos de habilidades (9 grupos), demandando sobre todo la identificación de características (Ic) y la descripción (Ds). Por su parte, solo tres grupos de españoles exigen dos tipos de habilidades (identificación de características (Ic) y descripción (Ds) o identificación de características (Ic) y justificación (J). Por último, un único grupo español y otro portugués exige tres tipos de habilidades, concretamente, la identificación de características (Ic), la descripción (Ds) y la explicación (Ex).

Tabla 2.

$N^{o}$ de habilidades que cada grupo de maestros en formación incluye en las cuestiones de evaluación sobre alimentación y salud.

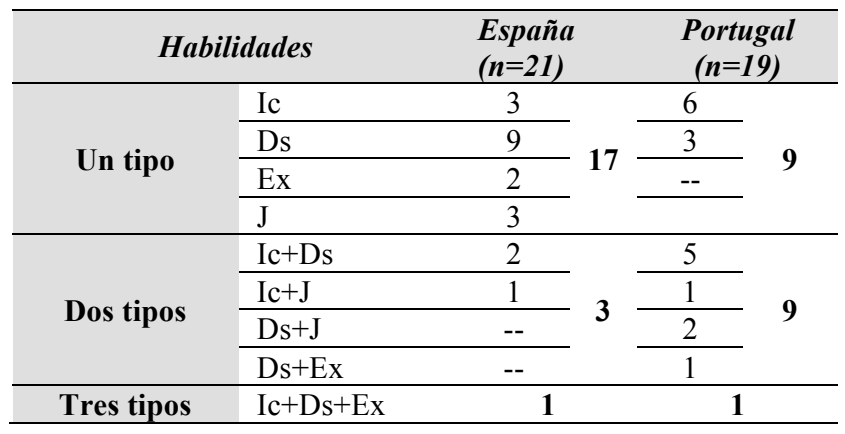

Analizamos a continuación las cuestiones concretas aportadas por los distintos grupos. Tal como se muestra en la tabla 3, las habilidades cognitivas, y más concretamente la identificación de características, se solicitan sobre todo a través de las ideas clave A2 (5 cuestiones en el caso español y 8 en el portugués- "Enumera los principales nutrientes"-) y A4 (4 cuestiones de españoles y 5 de portugueses - "Selecciona de los siguientes hábitos aquellos que consideres más saludables”-). 
Por su parte, en lo que se refiere a las habilidades cognitivo-lingüísticas, la descripción se exige mayoritariamente a través de la idea clave A2 en el caso español (12 cuestiones - ¿En qué consiste llevar una buena alimentación?”-) y, a mayores también a través de la idea A4 en el portugués (6 cuestiones en cada caso - “Cómo podemos evitar enfermedades alimenticias"-). Sin embargo, la explicación en el caso español se requiere tanto en relación a la idea clave A2 (3 cuestiones - "Explica por qué es necesario mantener una alimentación variada y equilibrada?”-) como A4 (3 cuestiones - "Explica qué le recomendarías a un paciente que está mal nutrido, si tú fueras un médico”-), mientras que en el potugués se solicita en la idea clave A3 (2 cuestiones - "Explica la relación entre alimentación y nutrición”-). Así mismo, la habilidad cognitivo-lingüística de justificación, se demanda en el caso español a través de la idea clave A2 (2 cuestiones - “Consideras que tu dieta es adecuada? ¿Por qué?”-), pero también a través de las ideas A5 (2 cuestiones - ¿Por qué unas personas o animales necesitan más alimento que otras? Justificalo"-) y A1 (1 cuestión - “En qué consiste llevar una alimentación saludable? Justificalo"-), mientras que en el caso portugués se demanda a través de la idea clave A4 (3 cuestiones - “Crees que los hábitos alimentarios influyen en nuestra salud? Explica por qué”-) y A3 (1 cuestión - “Justifica la relación entre alimentación y nutrición”-)

Tabla 3.

Relación entre las ideas clave y las habilidades solicitadas en cada pregunta planteada por los futuros maestros sobre alimentación y salud.

\begin{tabular}{|c|c|c|c|c|c|c|c|c|c|}
\hline & & \multicolumn{8}{|c|}{ Tipos de habilidades } \\
\hline & & \multirow{2}{*}{\multicolumn{2}{|c|}{$\frac{\text { Cognitivas }}{I c}$}} & \multicolumn{6}{|c|}{ Cognitivo-lingüisticas } \\
\hline & & & & \multicolumn{2}{|c|}{$D s$} & \multicolumn{2}{|c|}{$E x$} & \multicolumn{2}{|c|}{$J$} \\
\hline & & $\mathbf{E}$ & $\mathbf{P}$ & $\mathbf{E}$ & $\mathbf{P}$ & $\mathbf{E}$ & $\mathbf{P}$ & $\mathbf{E}$ & $\mathbf{P}$ \\
\hline \multirow{5}{*}{ 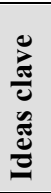 } & A1 & -- & 2 & 1 & 2 & 1 & -- & 1 & -- \\
\hline & $\mathbf{A 2}$ & 5 & 8 & 12 & 6 & 3 & -- & 2 & -- \\
\hline & $\mathbf{A 3}$ & -- & -- & -- & 1 & -- & 2 & -- & 1 \\
\hline & A4 & 4 & 5 & 4 & 6 & 3 & -- & -- & 3 \\
\hline & A5 & 2 & 1 & -- & 1 & -- & -- & 2 & -- \\
\hline
\end{tabular}

\section{Conclusiones}

Aunque los futuros maestros participantes en esta investigación, tanto los españoles como los portugueses, formulan cuestiones variadas referidas a la evaluación de distintos aspectos relativos a la alimentación y la salud, solo los portugueses plantean escasas cuestiones referidas a la relación de la alimentación saludable con la nutrición. En términos generales, los estudiantes se centran en evaluar aspectos referidos a las características de la alimentación saludable (aprendizaje de la dieta equilibrada, la pirámide nutricional...), siendo la relación entre alimentación variada menos demandada entre ambos colectivos, a lo que los españoles le suman a esa escasa demanda los hábitos saludables.

Si bien, a través de la evaluación, los maestros en formación, en general, demandan habilidades cognitivas (concretamente la identificación de características) y cognitivo-lingüísticas, estas últimas están especialmente centradas en la descripción, habilidades como la explicación y la justificación son muy poco consideradas.

Además, los distintos grupos de estudiantes españoles plantean mayoritariamente cuestiones referidas a una única idea clave, demandando habitualmente una única habilidad, mientras que los portugueses también suelen demandar dos tipos de habilidades, destacando la identificación de características y la descripción.

A pesar de que el número de participantes en este trabajo es reducido y los resultados deben tomarse con cautela, consideramos que las conclusiones de este estudio indican que, aunque es adecuado que los futuros maestros tengan pensado enseñar hábitos saludables relacionados con la alimentación, no deja de quedar incompleto si no se solicita paralelamente la justificación de dichos hábitos desde la teoría, empleando la relación existente entre la alimentación y la nutrición y favoreciendo así el desarrollo de la competencia científica (Cabello et al., 2016). Por otra parte, esta escasa solicitud de la justificación teórica de los hábitos saludables resulta coherente con la priorización de habilidades poco exigentes, como la identificación de características y la descripción, mientras que la necesaria justificación tiene muy escasa presencia en las cuestiones de evaluación, mostrando así una visión restringida sobre el tipo de conocimientos que deben adquirir los niños de Educación Primaria. Ello puede deberse a la influencia de la formación científica previa que han recibido los estudiantes. No podemos olvidar que la enseñanza de este tema y concretamente los libros de texto, ampliamente utilizados como soporte de la misma, suelen promover esa visión restringida anteriormente señalada (Rivadulla López, 2013).

Estos datos constituyen una llamada de atención para la formación docente, pues muestran la insuficiencia de ésta para cambiar prioridades en lo que a la selección de contenidos a evaluar se refiere. Aunque la introducción de la Educación para la Salud como materia optativa en los Planes de Estudio de las carreras de Magisterio de las universidades españolas es habitual y deseable, y sin duda favorece la formación docente, es importante que a través de ella se insista en qué contenidos enseñar y se complemente la enseñanza de la dieta equilibrada, hábitos saludables... con la relación entre la alimentación saludable y la propia función de la nutrición.

Para finalizar, hay que indicar que el conocimiento científico ha de movilizarse para explicar procesos concretos e interesantes para el alumnado. La dieta equilibrada y su conocimiento es esencial pues repercute en los hábitos saludables de la población (Del Carmen, 2010; Pérez de Eulate \& Llorente, 2015), pero además, también es importante por los problemas alimenticios acuciantes (sobrepeso, obesidad, anorexia, bulimia...) que está teniendo la sociedad actual en general y la población infantil en particular. Por ello, es importante que el alumnado no solo conozca la relación entre dieta equilibrada y los problemas alimenticios que acarrea dietas inadecuadas, sino que también los sepa justificar. 


\section{Referencias}

Banet, E., \& López, C. (2010). ¿Cómo mejorar el desayuno de los escolares de Educación Primaria? Investigación en la Escuela, 71, 63-83.

Blanco Loro, M. E. (2010). Políticas evaluativas del profesorado de Primaria, condicionantes de su actividad docente. Bordón, 62(1), 29-47.

Briceño, J. J., Benarroch, A., \& Marín, N. (2013). Coherencia epistemológica entre Ciencia, aprendizaje y enseñanza de profesores universitarios colombianos. Comparación de resultados con profesores chilenos y españoles. Enseñanza de las Ciencias, 31(2), 55-74.

Brown, G. T., Lake, R., \& Matters, G. (2011). Queensland teachers' conceptions of assessment: The impact of policy priorities on teacher attitudes. Teaching and Teacher Education: An International Journal of Research and Studies, 27(1), 210-220. doi: 10.1016/j.tate.2010.08.003

Cabello, A., España, E., \& Blanco, A. (2016). La competencia en alimentación. Barcelona: Octaedro.

Del Carmen, L. (2010). Los materiales de desarrollo curricular: un cambio imprescindible. Investigación en la Escuela, 72, 53-60.

Del Carmen, L. M. (2010). La alimentación: algo más que ingerir alimentos. Alambique, 66, 66-72.

García Barros, S., \& Martínez Losada, C. (2014). La importancia de las habilidades cognitivo-lingüísticas asociadas al estudio de la Astronomía desde la perspectiva del profesorado Enseñanza de las Ciencias, 32(1), 179-197.

Goytia, E., Besson, I., Gasco, J., \& Domènech, J. (2015). Evaluar habilidades científicas. Indagación en los exámenes. ¿Una vía para cambiar la práctica didáctica en el aula? Alambique, 79, 1-11.

Hernández, L. (2010). Evaluar para aprender: hacia una dimensión comunicativa, formativa y motivadora de la evaluación. Enseñanza de las Ciencias, 28(2), 285-293.

Jorba, J., Gómez, I., \& Prat, P. (2000). Hablar y escribir para aprender. Uso de la lengua en situación de enseñanza aprendizaje desde las áreas curriculares. Barcelona: ICE Universitat Autònoma de Barcelona. Síntesis.

López-Lozano, L., \& Solís-Ramírez, E. (2016). ¿Para qué, cómo y qué evalúa en ciencia el profesorado de Primaria en formación? Revista Eureka sobre Enseñanza y Divulgación de las Ciencias, 13(1), 102-120.

Martínez Losada, C., \& García Barros, S. (2013). Habilidades cognitivolingüísticas y nutrición humana en la formación inicial del profesorado de secundaria. Revista Eureka sobre Enseñanza y Divulgación de las Ciencias, 10(Extraordinario), 664-677.

McMillanm, J., \& Shumacher, S. (2005). Investigación educativa una introducción conceptual. Madrid: Pearson.

Miralles, P., Gómez, C. J., \& Sánchez, R. (2014). Dime qué preguntas y te diré qué evalúas y ense nas. Análisis de los exámenes de ciencias sociales en tercer ciclo de Educación Primaria. Aula Abierta, 42, 83-89.
Núñez, G., Mazzitelli, C., \& Vázquez, S. (2007). ¿Qué saben nuestros alumnos sobre alimentación y nutrición? Revista Iberoamericana de Educación, 5(43), 1-8.

Pérez de Eulate, L., \& Llorente, E. (2015). ¿Qué enseñar en la educación obligatoria acerca de la alimentación y la actividad física? Un estudio con expertos. Enseñanza de las Ciencias, 33(1), 85-100.

Prain, V. (2006). Learning from Writing in Secondary Science: Some theorical and practical implications. International Journal of Science Education, 28, 179-201.

Rivadulla López, J. C. (2013). El desarrollo del currículum desde la perspectiva del profesorado. La nutrición humana. Universidade da Coruña, A Coruña.

Rivadulla López, J. C., García Barros, S., \& Martínez Losada, C. (2013). La enseñanza de la nutrición humana en Educación Primaria desde una perspectiva medioambiental AmbientalMente Sustentable, 15-16(1), 19-29.

Rivarosa, S., \& De Longhi, A. (2006). La noción de alimentación y su representación en alumnos escolarizados. Revista electrónica de Enseñanza de las Ciencias, 5(3), 534-552.

Sabariego, M., Massot, I., \& Dorio, I. (2012). Métodos de Investigación Cualitativa. In J. Etxebarría \& J. Tejedor (Eds.), Metodología de la Investigación Educativa (pp. 293-328). Madrid: La Muralla.

Solís-Ramírez, E. (2012). A investigação na formação inicial do professorado: uma aproximação às concepções curriculares do professorado de Ciências de Educação Secundária. In J. O. Da Silva Uggioni (Ed.), Saberes Docentes (pp. 139-175). São Paulo: Iglu Editora.

Stake, R. (2007). A arte de investigação com estudos de caso. Lisboa: Fundação Calouste Gulbenkian.

Titrek, O., \& Cobern, W. W. (2011). Valuing Science: A Turkish-American comparison. International Journal of Science Education, 33(3), 401-421.

Vidgen, H. (2016). Food literacy: key concepts for health and education. Londres: Routledge.

Wang, J. R., Kao, H. L., \& Lin, S. W. (2009). Preservice teachers' initial conceptions about assessment of science learning: the coherence with their views of learning science. Teaching and Teacher Education, 26(3), 522-529.

Weurlander, M., Söderberg, M., Scheja, M., Hult, H., \& Wernerson, A. (2012). Exploring formative assessment as a tool for learning: Students' experiences of different methods of formative assessment. Assessment and Evaluation in Higher Education, 37(6), 747-760.

Young, S., \& Nehm, R. (2011). A Cross-Cultural Comparison of Korean and American Science Teachers' Views of Evolution and the Nature of Science. International Journal of Science Education, 33(2), 197-227. 
RIVADULLA-LÓPEZ, FUENTES-SILVEIRA, GARCÍA BARROS

\section{Agradecimiento}

La recogida de datos en Portugal se realizó durante una estancia postdoctoral de investigación en la Universidade do Minho, subvencionada por la Vicerreitoría de Política Científica, Investigación e Transferencia de la Universidade da Coruña.

Trabajo financiado por el Ministerio de Ciencia e Innovación EDU2011-27772. 